\title{
Bubble Pump Models Verification Based on the Experimental Results
}

\author{
Bella Gurevich \\ Shamoon College of Engineering, Mechanical Engineering Department \\ Jabotinsky 24, Ashdod, Israel \\ bellagu@sce.ac.il
}

\begin{abstract}
DAR) cycle is driven by heat and has no moving parts. It is based on refrigerant and absorbent as the working fluid together with hydrogen as an auxiliary inert gas. To circulate the working fluid without a mechanical pump, the diffusion absorption cycle relies on a bubble pump. Experimental system was designed and operated. Its aim was to investigate the performance of a bubble pump operating with three lifting tubes. The experimental results of the system were compared to existing models. The comparison showed that there was a bad agreement between the experimental and the theoretical results.
\end{abstract}

Keywords: bubble pump, DAR cycle, multiple lift tubes, binary solution

\section{Introduction}

A diffusion absorption refrigeration (DAR) cycle is driven by heat and has no moving parts. It is based on refrigerant and absorbent as the working fluid together with hydrogen as an auxiliary inert gas. To circulate the working fluid without a mechanical pump, the diffusion absorption cycle relies on a bubble pump. The bubble pump is a heated tube (length L and diameter D) communicating between a reservoir and a separating unit which uses heat for circulating the binary working fluid (refrigerant which has a lower boiling temperature than the organic solvent). Heat is applied at the bottom of the bubble pump at a rate sufficient to evaporate some of the refrigerant from the binary solution. The resulting vapor bubbles of the refrigerant rise in the tube carrying the liquid, poor solution, (which contains the solvent and the refrigerant that was not evaporated) to the higher reservoir. The desorbed gas is separated from the absorbent and flows to the condenser where it exchanges latent heat with the cold environment.

These systems are reliable, quiet and portable. Waste heat or solar radiation can be used in order to operate the system. The disadvantage of these systems is the low cooling capacities. One of the causes for the low cooling capacities values of such systems is the relatively small amounts of the separated refrigerant from the rich solution (mixture of solvent with high concentration of refrigerant). The bubble pump performance is determined by the amount of the separated refrigerant leaving the bubble pump and circulating ratio (i.e. the ration between the mass flow rate of the separated refrigerant and the mass flow rate of the rich solution).

This work is aimed to compare between experimental results of [1] and existing theoretical models that determine the amount of the separated refrigerant.

[2] developed a thermodynamic model that predicted a correlation between the submergence ratio and the flow rates of the two phase mixture for a single component two phase solution. The theoretical model used mass and momentum equations for two-phase flow with a binary solution, assuming slug flow regime and drift flux model. 


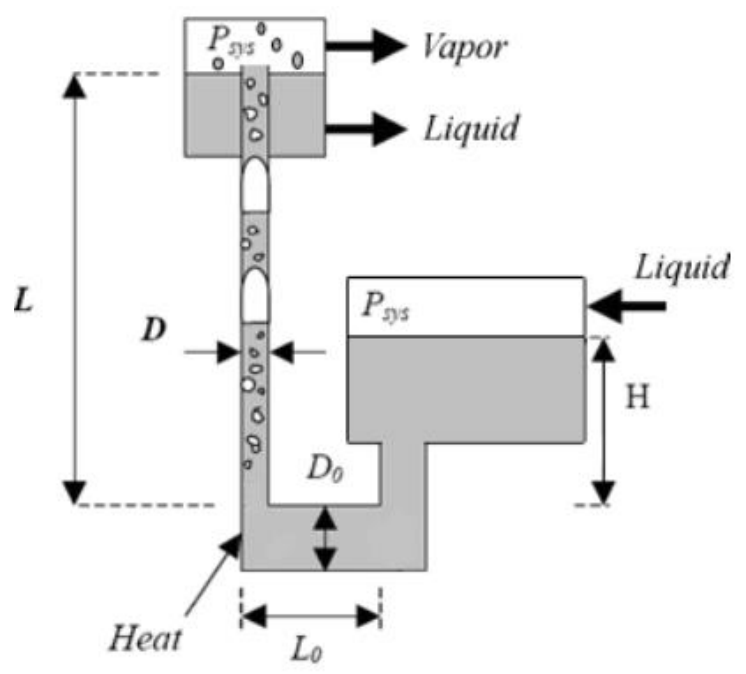

Fig. 1: Schematic drawing of Delano's model.

Frictional losses along the tubes were neglected as well as the momentum change in the bubble pump.

Expressing the submergence ratio $\frac{H}{L}$ in terms of volumetric flow rates $Q_{G}, Q_{L}$ gives an equation in form of:

$$
\frac{H}{L}=\frac{1}{1+\left(\frac{\dot{Q_{G}}}{Q_{L} \cdot s}\right)}+\frac{U_{L S}{ }^{2}}{2 \cdot g \cdot L}\left[K \cdot\left(1+\frac{\dot{Q_{G}}}{\dot{Q_{L}}}\right)^{2}+2 \cdot \frac{\dot{Q_{G}}}{\dot{Q_{L}} \cdot}+1\right]
$$

$\mathrm{K}$ was the friction factor and defined by:

$$
K=\frac{4 \cdot f_{T P} \cdot L}{D}
$$

Where $f_{T P}$ is the two phase friction factor. For Delano's system was found that $\mathrm{K}=17$.

[3] applied Deleno's model for calculating the bubble pump. He concluded that a correlation of a lose factor $\mathrm{K}$ is necessary for the model to account for the discrepancies observed between the actual experimental observations and the assumption made in the theoretical analysis. The correction of the lose factor is a function of vapor volumetric flow rate and the pump tube diameter as:

$$
K=A \cdot V_{\text {vap }}^{4}+B \cdot V_{\text {vap }}^{3}+C \cdot V_{\text {vap }}^{2}+D \cdot V_{\text {vap }}+E
$$

Where $\mathrm{A}, \mathrm{B}, \mathrm{C}, \mathrm{D}$ and $\mathrm{E}$ are parameters that are a function of the pump tube diameter and the vapor volumetric flow rate $V_{\text {vap }}$.

[4] performed both experimental and theoretical analysis on an air lift bubble pump at atmospheric conditions. The results showed that there were optimum operational conditions for a specific diameter and submergence ratio. From the experiments it was found that bubble pump operation is not sensitive to the length of the lift tube, however it was highly 
dependent on the submergence ratio $(\mathrm{H} / \mathrm{L})$. [5] presented a mathematical model to determine the bubble pump performance. An individual test was conducted on a simple air-water lift apparatus of a given configuration (10.9 mm pump tube inner diameter, 100mm lift head and $650 \mathrm{~mm}$ driving head), at atmospheric pressure. The obtained relation between liquid flow $V_{\text {liq }}$ and air flow $V_{\text {vap }}$ leaving the separator was:

$$
V_{\text {liq }}=-0.0004 \cdot V_{\text {vap }}^{4}+0.00625 \cdot V_{\text {vap }}^{2}+0.63772 \cdot V_{\text {vap }}-0.46802
$$

Based on previous experimental results by [2] and [4], it was found that slug flow pattern is the most suitable for optimal operation of the bubble pump, yet, it is limited by the diameter size of the tube where evaporation occurs. The aim of the experimental work was to test whether a change in the configuration of the generator influences the performance of the bubble pump, i.e. the amount of the desorbed refrigerant.

The geometrical configuration of the traditional single bubble pump pipe, which was commonly used in many previous works ([2] , [4] and [6].), was replaced by a modular set of parallel bubble pump pipes ([1]). Multiple lift tube configurations were experimentally investigated by [7], [8] and [1]. Their results indicated that an addition of lift tubes increased the pump's ability to handle larger heat loads and flow rates before the flow pattern changed from slug to annular.

\subsection{Experimental System}

A standard single bubble pump configuration was replaced by a set of parallel bubble pumps operating with an environmentally friendly solution of R134a-DMAC (consideration in choosing the working fluids was based on work done by [9]). The design of the systems allowed changes to the heat input, the motive head and enabled insertion of additional R134a to change the concentration of the solution.

The experimental system is lacking an evaporator, a condenser, and the inert gas, since the focus only on the performance of the bubble pump, the system is not considered a complete diffusion absorption cooling system. The system comprise a reservoir, a heating unit (generator), a separation unit (separator), and an absorption unit, all of which are schematically illustrated in Fig.1. In the experimental system designed by the rich solution in the reservoir flows downwards to the heating unit. Due to unique structure of the generator, the heated rich solution splits into three vertical lifting tubes. The three lifting tubes are connected to the exit of the generator where the heat for refrigerant evaporation is supplied by a manually operated variac powered by an electrical DC current. Vertical flow is driven by the evaporated refrigerant bubbles, which carry the liquid solution up the lifting pipes to the separation unit. The experimental system is not modular, since there is one rich solution pipe connected to the generator's entrance. The separator's special doubleoutlet design enables upward flow of the gaseous refrigerant, and downward flow of the liquid poor solution. The liquid binary solution contains solvent and refrigerant that is not separated during evaporation (poor solution). The amount of separated refrigerant defines bubble pump performance. Both the hot refrigerant and the hot poor solution flow through bent copper tubes where they are cooled by heat exchange with the environment. The pipes for refrigerant and poor solution then merge into a single pipe, resulting in absorption of the refrigerant in the poor solution, a reaction accompanied by an exothermic effect, and further heat exchange with the environment. The combined solution of refrigerant and poor solution then flows back into the reservoir. The inclusion of metering devices in the system makes it possible to know the temperature, pressure, and flow rate at points of interest in real time. 


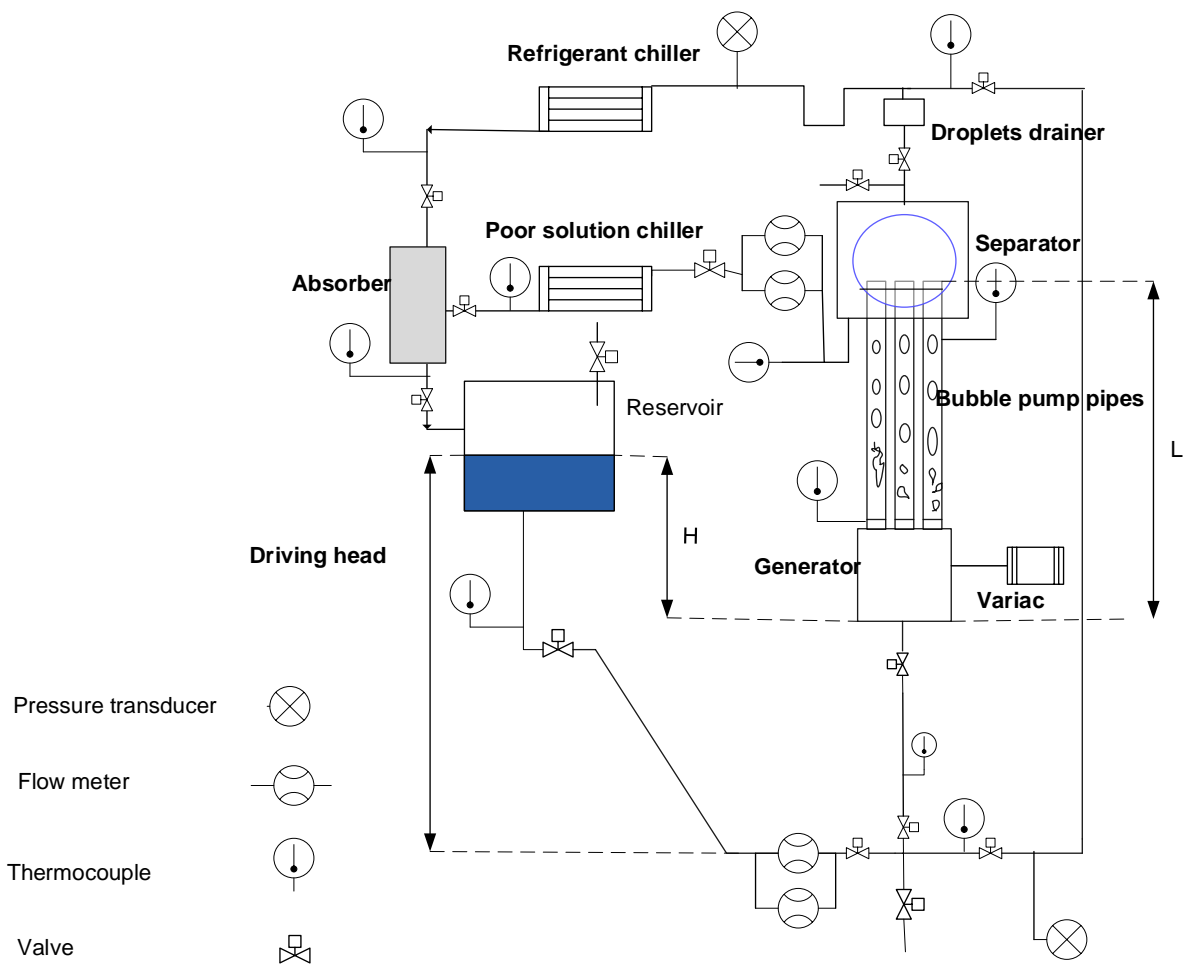

Fig. 2: Layout of the experimental system.

\subsection{Experimental Results}

Delano's model [2] and the experimental results are compared and from Fig. 3 it show shows that there is not a good agreement between the values of the void fraction.

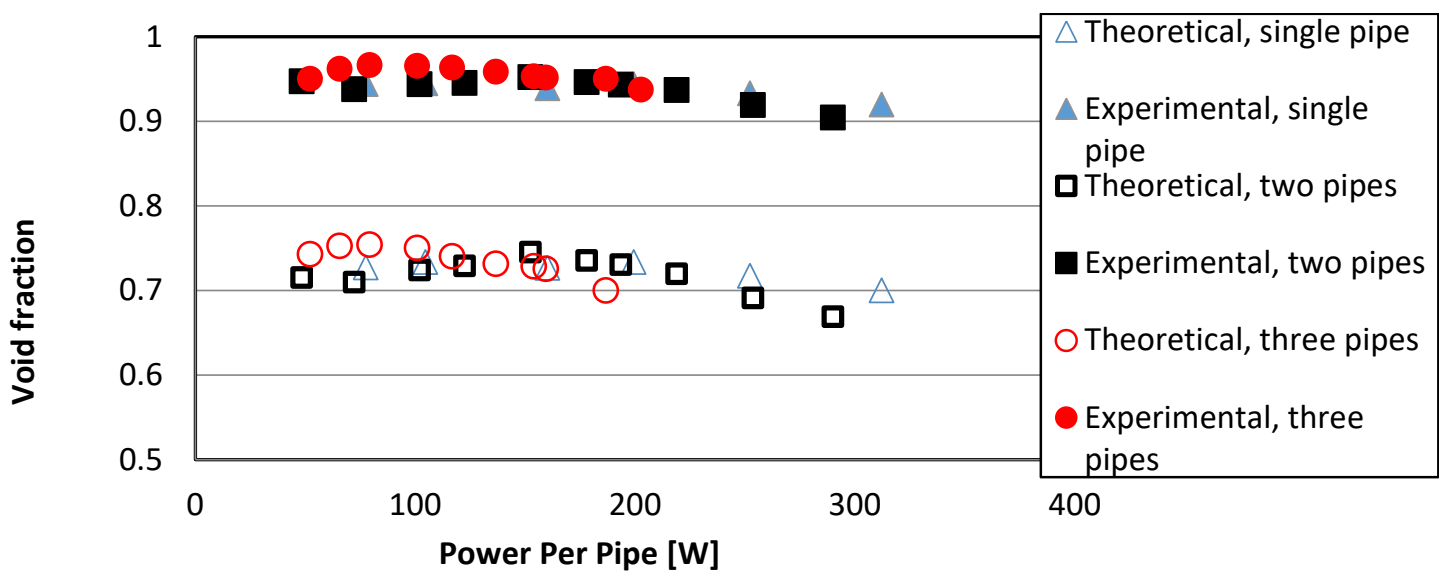

Fig. 3: void fraction vs. supplied heating power per pipe.

Comparison between the experimental results and the [5] model shows that there is not a good agreement with model's results. The system's $4^{\text {th }}$ order polynomial (that describes the connection between the poor solution volumetric flow rate and the refrigerant volumetric flow rate) has completely different values of coefficients.

HTFF 113-4 


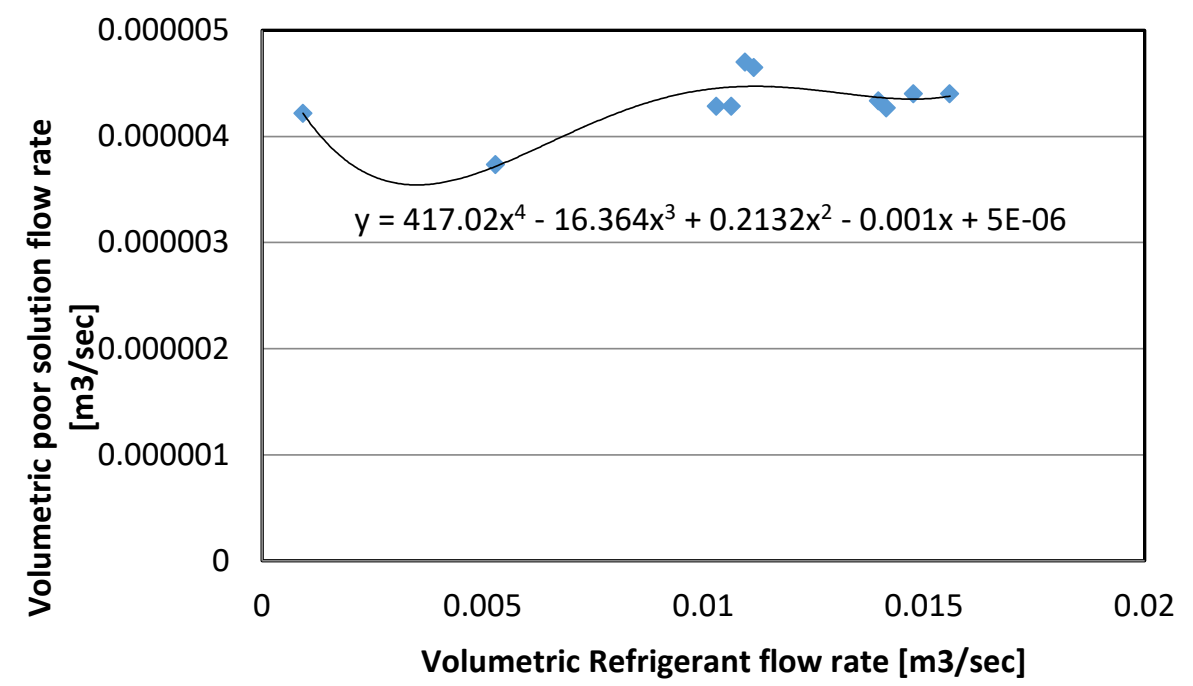

Fig. 4: Comparison between the experimental results.

\section{Conclusions}

The differences between the Delano model [2] and the current experimental results as well as the difference between Pongsid and Satha model [5] are due to the models' assumptions. The models do not take in considerations that, during the process of lifting there is a back absorption of the refrigerant into the solution. Also the models assumes slug flow regime along the pipe. Slug collisions and acceleration are not considered as well. Note that the liquid phase in this work is a binary solution. Along the bubble pump, as the two phase solution exits the generator, it is not in equilibrium until the solution reaches the separator.

\section{References}

[1] B. Gurevich, M. Jelinek, A. Levy, I. Borde, "Performance of a set of parallel bubble pumps operating with a binary solution of R134a-DMAC," Applied Thermal Engineering, vol. 75, pp. 724-730, 2015.

[2] A. D. Delano, "Design analysis of the Einstein refrigeration cycle Robot Vision," Ph.D. thesis, Georgia Institute of Technology, 1998.

[3] A. Sathe, "Experimental and Theoretical Studies on a Bubble Pump for a Diffusion-Absorption Refrigeration System," M.S. thesis, Indian Institute of Technology Madras and University of Stuttgart, 2001.

[4] S. J. White, "Bubble pump design and performance," Master's thesis, Georgia Institute of Technology.

[5] S. Pongsid, A. Satha, "Investigation of A Diffusion Absorption Refrigerator," Dep. Mech. Eng. Srindhor. Inter.Inst. of Technology, Thiland. 2002.

[6] A. Koyfman, M. Jelinek, A. Levy, I. Borde, "An experimental investigation of bubble pump performance for diffusion absorption refrigeration system with organic working fluids," Applied Thermal Engineering, pp. 18811894, 2003.

[7] G. Vicatos, A. Bennett, "Multiple lift tube pumps boost refrigeration capacity in absorption plants," Journal of Energy in Southern Africa, vol. 18, no. 3, pp. 49-57, 2007.

[8] H. Monsef, M. Naghash Zadegan, K. Javaherdeh, "Design and Construction of a Low Capacity Pump-Less Absorption System," Mechanical Engineering Department, University of Guilan, Iran, 2012.

[9] I. Borde, M. Jelinek, N. C. Daltrophe, "Development of Advanced Absorption Systems Driven by Low Temperature Heat Sources," Int. J. Refrigeration, vol. 31, pp 521-530, 1993. 